\title{
Design of High-C Cr-Co-Ni medium Entropy Alloy for Tribological Applications ${ }^{\dagger}$
}

\author{
Gustavo Bertoli ${ }^{1}$, Guilherme Y. Koga ${ }^{2}$, Fernanda C. Puosso ${ }^{2}$, Amy J. Clarke ${ }^{3}$, Claudio S. \\ Kiminami ${ }^{2}$ and Francisco G. Coury ${ }^{2}$ \\ 1 Graduate Program in Materials Science and Engineering, Federal University of São Carlos, Rod. Washington \\ Luis, km 235, São Carlos, SP, Brazil \\ 2 Department of Materials Science and Engineering, Federal University of São Carlos, Rod. Washington Luis, \\ km 235, São Carlos, SP, Brazil \\ 3 George S. Ansell Department of Metallurgical and Materials Engineering, Colorado School of Mines, 1500 \\ Illinois St., Golden, CO, USA \\ + Presented at the Entropy 2021: The Scientific Tool of the 21st Century, 5-7 May 2021; Available online: \\ https://sciforum.net/conference/Entropy2021/.
}

Published: 5 May 2021

Medium and high entropy alloys (MEAs/HEAs), which typically have three or more main elements, were initially designed to have high configurational entropy ( $\left.S_{\text {conf }}\right)$ stabilizing a simple, single-phase solid solution (SS) over multi-phase alloys. Having multiple main elements will indeed increase the $S_{\text {conf }}$ of simple SSs, but it may decrease the enthalpy also increase, in a lower degree, Sconf of other complex phases, leading to multi-phase microstructures. Therefore, although the initial idea behind MEAs/HEAs was refuted, the interest remains, the focus shifted to exploring its vast compositional field, which includes an almost infinite number of alloys. Some of them with potentially better properties than those existing today. The Cantor alloy (equiatomic $\mathrm{CrMnFeCoNi)}$ and the equiatomic $\mathrm{CrCoNi}$ alloy, both single-phase with face-centered cubic (FCC) structure, are among the toughest materials ever reported. In this work, computational thermodynamic calculations (CALPHAD method) predicted that $\mathrm{C}$ additions in the $\mathrm{Cr}_{40} \mathrm{CO}_{40} \mathrm{Ni}_{20}$ MEA favor the formation of a promising multiphase microstructure for wear applications. A large amount of $C$ was incorporated into the alloy by melting in a graphite crucible, this process allowed $C$ saturation in the melt, which as will be shown, can be well controlled by carefully selecting the casting temperature, in this case we achieved 24 at $\%$ C

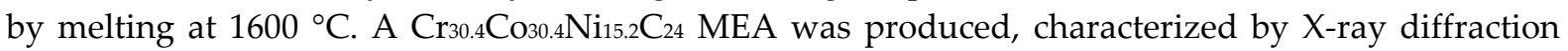
(XRD) and scanning electron microscopy (SEM) equipped with energy-dispersive spectroscopy (EDS). Experimental results revealed a microstructure composed of: graphite flakes, hard primary Cr-rich carbides, and a tough eutectic matrix of FCC phase and carbides; in good agreement with thermodynamic calculations. These findings highlight the great flexibility and potential in MEAs/HEAs design, making it possible to obtain microstructures and sets of properties that are beneficial for a given application.

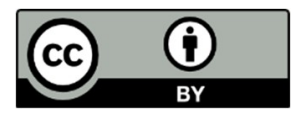

(C) 2021 by the authors. Licensee MDPI, Basel, Switzerland. This article is an open access article distributed under the terms and conditions of the Creative Commons Attribution (CC BY) license (http://creativecommons.org/licenses/by/4.0/). 